\title{
Plasmid transferability of KPC into a virulent K2 serotype Klebsiella pneumoniae
}

Leung-Kei Kristopher Siu ${ }^{1,2}$, David B Huang ${ }^{3,5^{*}}$ and Tom Chiang ${ }^{3,4}$

\begin{abstract}
Background: KPC-producing carbapenem-resistant Klebsiella pneumoniae (CRKP) infections are associated with high mortality; however, their virulence determinants are not well defined.

Methods: We investigated the virulence and plasmid transferability among KPC-containing K. pneumoniae isolates. Results: KPC-2 and -3 were successfully conjugated and retained by a virulent K2 K. pneumoniae recipient isolate. Antimicrobial susceptibility testing showed KPC-2 and -3 donor strains were resistant to more than four classes of antibiotics while the K2 isolate was only initially resistant to ampicillin. After conjugation of KPC-2 and -3 , the K2 K. pneumoniae transconjugants became resistant to all beta-lactams. Additionally, the KPC K2 K. pneumoniae transconjugants continued to retain its high serum resistance and murine lethality.

Conclusions: Conjugation and retainment of KPC by virulent K2 K. pneumoniae and the ability of the tranconjugants to maintain its high serum resistance and murine lethality after conjugation was demonstrated in this study. These findings are concerning for the potential of KPC-like genes to disseminate among virulent K. pneumoniae isolates.
\end{abstract}

Keywords: Klebsiella pneumoniae, Virulence, Plasmid mediated resistance

\section{Background}

In the Asia Pacific Rim, a pathogen that is well documented is the hypervirulent (hypermucoviscous) variant of Klebsiella pneumoniae. This pathogen causes unique clinical invasive syndromes of community-acquired $K$. pneumoniae infections including pyogenic liver abscess and a propensity for metastatic spread to distant sites in the absence of a history of hepatobiliary disease [1-3]. In Western countries, the predominant infections due to K. pneumoniae are still the "classic" strains. Infections caused by $K$. pneumoniae can result in serious and life threatening infections including pneumonia, urinary tract infections, intravascular line infections, soft tissue infections, intraabdominal infections and bacteremia. Because carbapenems are usually the last line of defense for the treatment of resistant Gram-negative pathogens, the development of carbapenemase-producing $K$. pneumoniae is

\footnotetext{
* Correspondence: dhuang82@hotmail.com

${ }^{3}$ Division of Infectious Diseases, Department of Medicine, Rutgers New Jersey Medical School, Newark, NJ, USA

${ }^{5}$ Rutgers New Jersey Medical School, 185 South Orange Avenue, Newark, New Jersey 07101, USA

Full list of author information is available at the end of the article
}

of great concern. Carbapenem-resistant $K$. pneumoniae (CRKP) has become a major threat and burden to healthcare systems worldwide as most isolates are resistant to multiple classes and some to all classes of antibiotics making treatment difficult if not near impossible. However, most of these KPC-containing strains especially in the United States do not contain the virulence factors of the strains found in Asia resulting in a less invasive clinical presentation. Likewise, virulent strains of $K$. pneumoniae causing invasive disease that are usually found in Asia are not known to be multi-drug resistant let alone carbapenem-resistant. However, there have been recent separate reports of endemic KPC K. pneumoniae strains and hypervirulent invasive $K$. pneumoniae strains found in New Jersey. This study assesses the possibility of an invasive virulent $\mathrm{K} 2 \mathrm{~K}$. pneumoniae strain and its capability of acquiring $\mathrm{KPC}$.

\section{Methods}

\section{Strains in this study}

The two most commonly found KPC strains (KPC-2 and -3 ) at the Veterans Affairs New Jersey Healthcare 
System (VA NJHCS) were used as donors. A virulent K2 serotype KP isolate from a liver abscess patient in New Jersey (University Hospital- Rutgers New Jersey Medical School) was chosen as the recipient [4]. These KPC KP donor isolates were acquired from the clinical microbiology laboratory at the VA NJHCS for this Institutional Review Board (IRB) approved study (MIRB 00937).

\section{Susceptibility testing}

Antimicrobial susceptibility was determined by the broth microdilution method [5], according to the Clinical and Laboratory Standards Institute's (CLSI) guidelines published in 2011. The following antimicrobial agents were used: cefazolin, amoxicillin/clavulanic acid, cefoxitin, cefotaxime, ceftazidime, imipenem, amikacin, gentamicin, ciprofloxacin, and trimethoprim-sulfamethoxazole.

\section{Pulsed-field gel electrophoresis}

Pulsed-field gel electrophoresis (PFGE) was performed as previously described [6]. The resulting genomic DNA profiles, or fingerprints, were interpreted according to established guidelines $[7,8]$.

\section{Confirmation of KPC production and KPC types by sequencing}

All isolates selected were confirmed for KPC production by polymerase chain reaction (PCR). A gene-specific primer set, KPC-F (5' ${ }^{\prime}$ ATG TCA CTG TAT CGC CGT CT- $\left.3^{\prime}\right)$ and KPC-R ( $5^{\prime}$-TTT TCA GAG CCT TAC TGC CC-3'), was used to determine the presence of the KPC $\beta$-lactamase sequence [9]. The amplicons (893 bp) were sequenced using an automated sequencer by the Sanger method (ABI Prism 377 sequencer; Perkin-Elmer) [10]. The generated sequences were compared to the National Center for Biotechnology Information (NCBI) database at www.ncbi.nlm.nih.gov/blast/.

\section{Conjugation experiments}

The transconjugant of the two most commonly found KPC strains (KPC-2 and -3) at the VA NJHCS, containing the KPC plasmid, were used as donors. The virulent K2 serotype Klebsiella pneumoniae (NJ-K2) isolate from a liver abscess patient in New Jersey was chosen as the recipient [4]. Inducing streptomycin resistance as a selection maker in the recipient for conjugation was performed according to previously described methods by Tasi et al. [11]. Briefly, the K2 serotype $K$. pneumoniae strain was grown in $\mathrm{LB}$ broth at $37^{\circ} \mathrm{C}$ to late-exponential growth phase and then spread on LB agar plates supplemented with $500 \mu \mathrm{g} / \mathrm{ml}$ streptomycin. The cells were incubated at $37^{\circ} \mathrm{C}$ and spontaneous mutants were obtained from colonies that arose within 3 days. This isolate possesses intrinsic resistance only to ampicillin and streptomycin. Mating procedure with $\mathrm{KPC}-2$ and -3 containing
KPC was then performed as previously described [12,13] except by using specific selection brilliant green agarcontaining inositol-nitrate-deoxycholate (BIND) for $K$. pneumoniae [14]. Serotype K2/KPC transconjugants were selected using the Klebsiella-selective medium BIND supplemented with $2 \mu \mathrm{g} / \mathrm{ml}$ of imipenem and $50 \mu \mathrm{g} / \mathrm{ml}$ of streptomycin. The virulent K2 serotype KPC K. pneumoniae transconjugant was able to grow on the selective BIND medium with imipenem. Positive transconjugants were confirmed by positive PCR with specific primer sequences to serotype K2 [15] and KPC type $\beta$-lactamases genes [16]. Transconjugants from the conjugation experiments were subcultured onto MacConkey or Mueller Hinton agar (BBL) for 14 days to check for KPC plasmid stability in KPC strains (KPC-2 and -3 ) and $K$. pneumoniae after conjugation (Figure 1).

\section{PCR detection of virulence-associated genes rmpA and aerobactin, and serotypes K1, K2, and K5}

PCR experiments to determine the presence of the specific genes for serotype $\mathrm{K} 1, \mathrm{~K} 2$, and $\mathrm{K} 5$; rmpA; and the aerobactin gene were performed as previously described $[15,17]$.

\section{Multilocus sequence typing (MLST)}

MLST were performed according to Turton et al. [18]. Sequences of seven housekeeping genes were obtained for isolates from liver abscess patients and carriers. Sequence information was compared with that available from the MLST website (http://pubmlst.org/kpneumoniae/) developed by Keith Jolley. Alleles and sequence types (STs) were assigned accordingly. Sequences of any alleles that were not in the database were submitted to the curator and a new allele number obtained. A difference in two or more alleles was considered to indicate that the sequence types being compared were unrelated.

\section{Virulence studies}

In vitro and in vivo assays to assess virulence were performed by serum resistance, neutrophil phagocytosis and mice lethality experiments. Neutrophil phagocytosis was performed as previously described [19]. The recipient serotype K2 K. pneumoniae (NJ-K2) isolated from a liver abscess was used as a control strain [4]. This strain is hypervirulent with a median lethal dose $(\mathrm{LD} 50)<10^{2}$ colony forming unit (CFU) and is highly phagocytic resistance ( $<10 \%$ of phagocytic rate 15 minutes).

For the determination of LD50 in mice, pathogen-free, adult, 6-8-week-old, male BALB/c mice weighing 20-25 g were obtained from the National Laboratory Animal Center. This study was carried out in strict accordance with the recommendations in the Guide for the Care and Use of Laboratory Animals of the National Institutes of Health. All animal care procedures and protocols were 


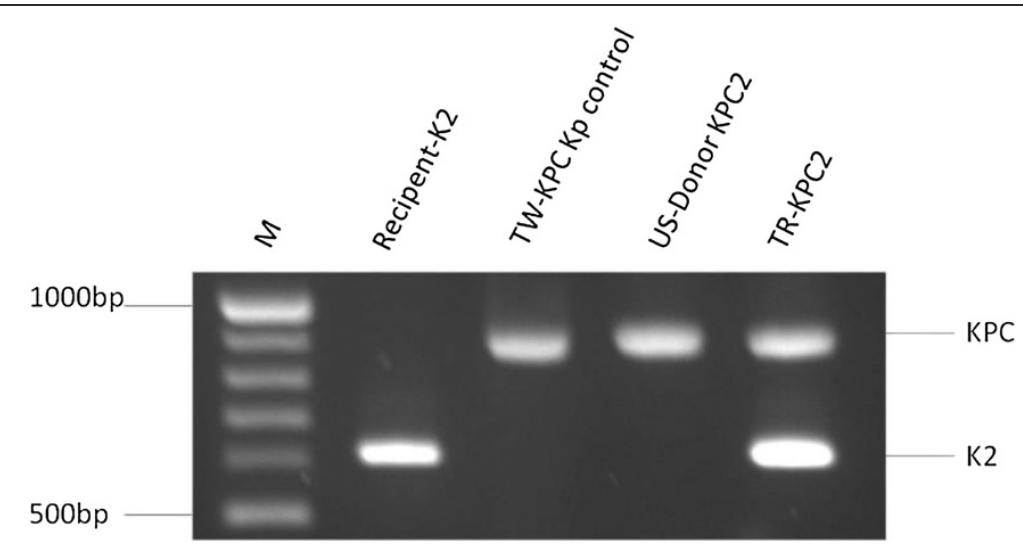

Figure 1 Conjugation experiments of plasmids carrying $\beta \mathrm{la}_{\mathrm{KPC}}-2$ and -3 from parental non-serotype $\mathrm{K} 2 \mathrm{~K}$. pneumoniae to recipient serotype K2 K. pneumoniae. In this conjugation experiment, plasmids carrying blaKPC from KPC-positive K. pneumoniae (KPC-2 and -3) were successfully transferred into the invasive $\mathrm{K} 2 \mathrm{~K}$. pneumoniae recipient isolate $(\mathrm{NJ}-\mathrm{K} 2$ ). Specifically, DNA extracted from the invasive K2 K. pneumoniae recipient isolate (NJ-K2) were positive for the blaKPC gene by PCR. Transconjugants, K2 K. pneumoniae::BlakPC, were obtained by Klebsiella-selective medium BIND supplemented with $2 \mu \mathrm{g} / \mathrm{ml}$ of imipenem. Colonies were then randomly selected from the plate and subjected for serotype K2 PCR. Colonies with PCR positive results on serotype determination were sub-cultured onto the same selective agar plate. Sub-culture of K2 K. pneumoniae:: $\beta$ lakPC Occurred by inoculating onto MacConkey agar plates for 14 consecutive days. Lane 1, PCR verification of recipient serotype K2 isolate from New Jersey; Lane 2, Control KPC-2 from Taiwan; Lane 3, donor K. pneumoniae with Bla $\mathrm{KPC}_{2}$, Lane 4, transconjugants K. pneumoniae::ßla $\mathrm{K}_{\mathrm{KPC}-2}$.

approved by the Institutional Animal Care and Use Committee of the National Health Research Institute (NHRI-IACUC-096004-A). Tenfold serial dilutions of KP were made from a starting concentration of $10^{6} \mathrm{CFU}$, and the adult $\mathrm{BALB} / \mathrm{c}$ mice had intraperitoneal injections with $0.1 \mathrm{ml}$ of each concentration. Six mice were used as a sample population for each bacterial concentration [20]. Signs and symptoms of infection were observed for 14 days.

Survival of the inoculated mice was recorded, and the LD50 was calculated using SigmaPlot version 7.0 from SPSS Inc.

\section{Results}

Conjugation of plasmids of KPC-2 and -3 into serotype recipient $\mathrm{K} 2$ and antimicrobial susceptibility test of their transconjugants

In the conjugation experiment, plasmids carrying blaKPC from KPC-positive $K$. pneumoniae (KPC-2 and -3) were successfully transferred into the invasive $\mathrm{K} 2 \mathrm{~K}$. pneumoniae recipient isolate (NJ-K2) (Figure 1). Subculture of serotype K2 K. pneumoniae::blaKPC onto selective agar plate showed good growth in the selective BIND agar plate. No plasmid loss after 14 days of subculture onto the MacConkey or Mueller Hinton agar plate was observed for K2 K. pneumoniae::blaKPC indicating the stability of the blaKPC plasmid in K. pneumoniae. All strains were verified by PCR to ensure that no contamination occurred during each experiment for donor, recipient of serotype K2 K. pneumoniae, or transconjugants K2 K. pneumoniae::blaKPC.
The KPC-positive K. pneumoniae (KPC-2 and -3) isolates showed $100 \%$ resistance to piperacillin-tazobactam, cefazolin, cefotaxime, ceftazidime, ceftriaxone, ciprofloxacin, cefoxitin, gentamicin, imipenem and/or meropenem. All $\beta$-lactam resistance were maintained in transconjugants while change of susceptibilities from resistance to susceptible were observed in aminoglycosides and quinolones indicating non-plasmid encoding resistance in these classes of antibiotics (Table 1).

\section{Detection of virulence-associated determinants and mice} lethality experiments

Detection of K1, K2, and K5 virulence-associated serotypes, and $r m p A$ and aerobactin genes, revealed that $\mathrm{KPC}$ donor isolates were non-K1, -K2, or - K5 serotypes and did not carry rmpA and aerobactin virulence genes. MLST showed that both donor isolates were ST-258 and recipient serotype K2 was ST-65. Transconjugants carrying plasmid encoding KPC-2 or 3 showed an increasing resistance to neutrophil phagocytosis in comparing to donor strains which carry KPC-2 or -3 carbapenemase (Figure 2). In assessing compliment killing by serum resistance assay, all the transconjugants were resistant to serum killing as recipient serotype $\mathrm{K} 2 \mathrm{~K}$. pneumoniae (Figure 3).

Murine lethality studies by IP injection showed that LD50 were $>10^{6} \mathrm{CFU}$ for the KPC K. pneumoniae isolates prior to conjugation, indicating low virulence. In contrast, LD50 of the transconjugant strain was $<10^{2} \mathrm{CFU}$ after conjugation while retaining KPC and $\mathrm{K} 2$ genes [confirmed by PCR (Figure 4)]. In order to confirm the stability 
Table 1 Antimicrobial susceptibility test among donors of KPC- 2 or -3 , recipient and transconjugant

\begin{tabular}{lllllll}
\hline Antibiotics & Recipient K2 & Donor & & \multicolumn{2}{c}{ Transconjugant } \\
\cline { 6 - 7 } & & KPC-2 & KPC-3 & & KPC-2 & KPC-3 \\
\hline Ampicillin & $\geq 32$ & $\geq 32$ & $\geq 32$ & $\geq 32$ & $\geq 32$ \\
Cefazolin & $\leq 2$ & $\geq 32$ & $\geq 32$ & $\geq 32$ & $\geq 32$ \\
Cefoxitin & $\leq 4$ & $\geq 128$ & $\geq 128$ & $\geq 128$ & $\geq 128$ \\
Aztreonam & $\leq 1$ & $\geq 32$ & $\geq 32$ & $\geq 32$ & $\geq 32$ \\
Cefotaxime & $\leq 1$ & 32 & 32 & 8 & 8 \\
Ceftazidime & $\leq 1$ & $\geq 32$ & $\geq 32$ & $\geq 32$ & $\geq 32$ \\
Cefepime & $\leq 1$ & $\geq 32$ & 16 & 16 & 8 \\
Ertapenem & $\leq 0.25$ & $\geq 8$ & $\geq 8$ & $\geq 8$ & $\geq 8$ \\
Imipenem & 0.5 & $\geq 8$ & $\geq 8$ & $\geq 8$ & $\geq 8$ \\
Meropenem & $\leq 0.25$ & $\geq 8$ & $\geq 8$ & $\geq 8$ & $\geq 8$ \\
Doripenem & $\leq 0.12$ & $\geq 4$ & $\geq 4$ & $\geq 4$ & $\geq 4$ \\
Colistin & 1 & $\leq 0.5$ & $\geq 4$ & $\leq 1$ & 1 \\
Tigecycline & $\leq 0.25$ & 1 & $\leq 0.25$ & $\leq 0.25$ & $\leq 0.25$ \\
Gentamicin & $\leq 1$ & $\leq 1$ & $\geq 16$ & $\leq 1$ & $\leq 1$ \\
Amikacin & $\leq 4$ & 16 & 32 & $\leq 4$ & $\leq 4$ \\
Ciprofloxacin & 0.12 & $\geq 4$ & $\geq 4$ & $\leq 0.06$ & 0.12 \\
Levofloxacin & $\leq 0.5$ & $\geq 8$ & $\geq 8$ & $\leq 0.5$ & $\leq 0.5$ \\
SXT* & $\leq 0.5$ & $\geq 4$ & $\geq 4$ & $\geq 4$ & $\leq 0.5$ \\
\hline Cotimoxazo & & & & &
\end{tabular}

*Cotrimoxazole.

of KPC-2 or 3 in transconjugants, PCR detection for serotype $\mathrm{K} 2$ and $\mathrm{KPC}-2$ or 3 were used during the infection period. PCR of serotype K2 and KPC-2 or 3 were detected on day 2 and day 3 of isolation indicating the KPC-2 or -3 plasmids were stable in the transconjugants during infection.

\section{Discussion}

KPC K. pneumoniae was first reported in the United States in 2001. Since its first report, KPC K. pneumoniae has become an increasingly prevalent pathogen and is now endemic in the Northeastern United States. Its spread has been well documented worldwide. According to data from the National Nosocomial Infections Surveillance System Report and the National Healthcare Safety Network, the proportion of Enterobacteriaceae that are carbapenem-resistant has increased from 1.2\% in 2001 to $4.2 \%$ in 2011. At the VA NJHCS and its affiliated University Hospital (Rutgers New Jersey Medical School), K. pneumoniae is now the second most prevalent Gram-negative pathogen with carbapenem resistance rates of $18 \%$ and $12 \%$ respectively (2012 VA NJHCS and University Hospital antibiograms). Most of the CRKP isolates found at these two hospitals are multi-drug resistant if not pan resistant to all available antibiotics. Not surprisingly, mortality rates of up to $50 \%$ have been reported in CRKP bacteremia cases due to the lack of effective antibiotics. Fortunately, CRKP has yet to be associated with community acquisition.

Invasive KP was initially thought of as a regionally distributed disease found in the Asia-Pacific Rim. However, it is now been reported worldwide as an emerging infectious disease [21]. Its virulence determinants and phagocytic inhibition has been well studied [22]. Unlike invasive $K$. pneumoniae, virulence and plasmid transmissibility of KPC K. pneumoniae have not been well investigated. Our previous study of virulence in KPC $K$. pneumoniae showed that in 60 unique CRKP, none had the virulent serotypes $K 1, K 2$, or $K 5$ nor the virulence factors of rmpA or aerobactin lending to the assumption that high rates of morbidity and mortality due to KPC K. pneumoniae are most likely due to its antibiotic resistance properties and not its virulence.

This study showed that the combination of an increased virulent strain of $K$. pneumoniae acquiring KPC and its associated multidrug associated genes can be created. Conjugation and retainment of KPC by virulent $\mathrm{K} 2 \mathrm{~K}$. pneumoniae and the ability of the tranconjugants to maintain its high serum resistance and lethality after

\section{Phagocytosis}

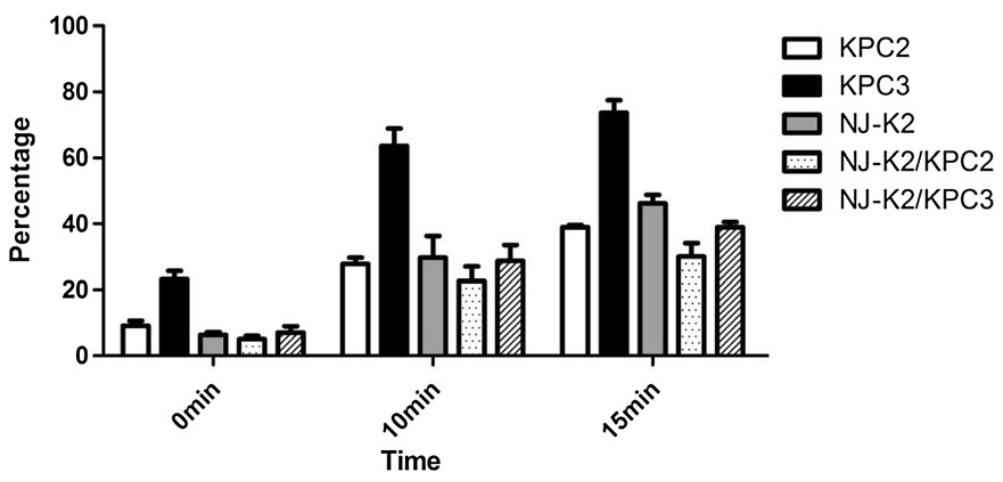

Figure 2 Neutrophils phagocytosis assays among Isolates of KPC-2 and -3 , serotype K2 recipient and their transconjugants. 


\section{Serum resistance}

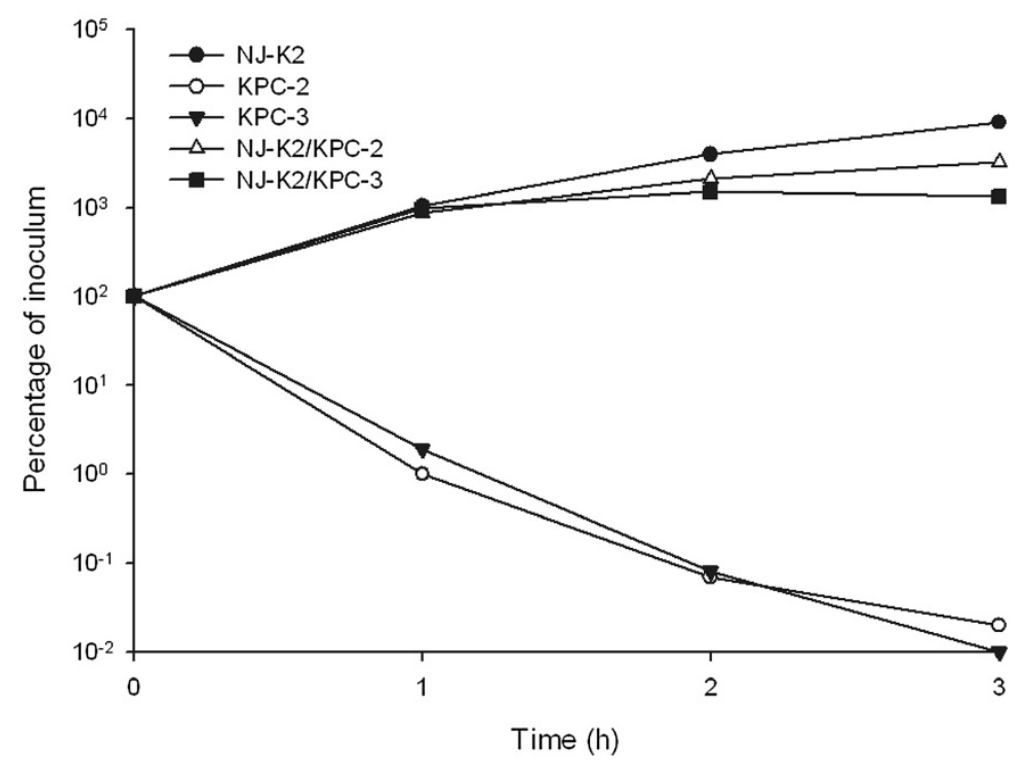

Figure 3 Serum resistance assays among Isolates of KPC-2 and -3 , serotype K2 recipient and their transconjugants.

conjugation were possible. The ramifications of having a virulent, multi-drug resistant, KPC-containing $K$. pneumoniae would be disastrous on healthcare systems [23]. Not surprisingly, with continued indiscriminate use of antibiotics worldwide, KPC-producing K. pneumoniae has been detected in China with the predominate clone being ST11 which is unrelated to the current ST258 clone found commonly in KPC K. pneumoniae found in the United States. With the continued worldwide dissemination of KPC, it is likely a matter of time before the virulent Asian strains of KP will begin to express KPC carbapenem resistance.

\section{Conclusions}

As demonstrated in our study, further dissemination of the KPC plasmid into virulent types of K. pneumoniae

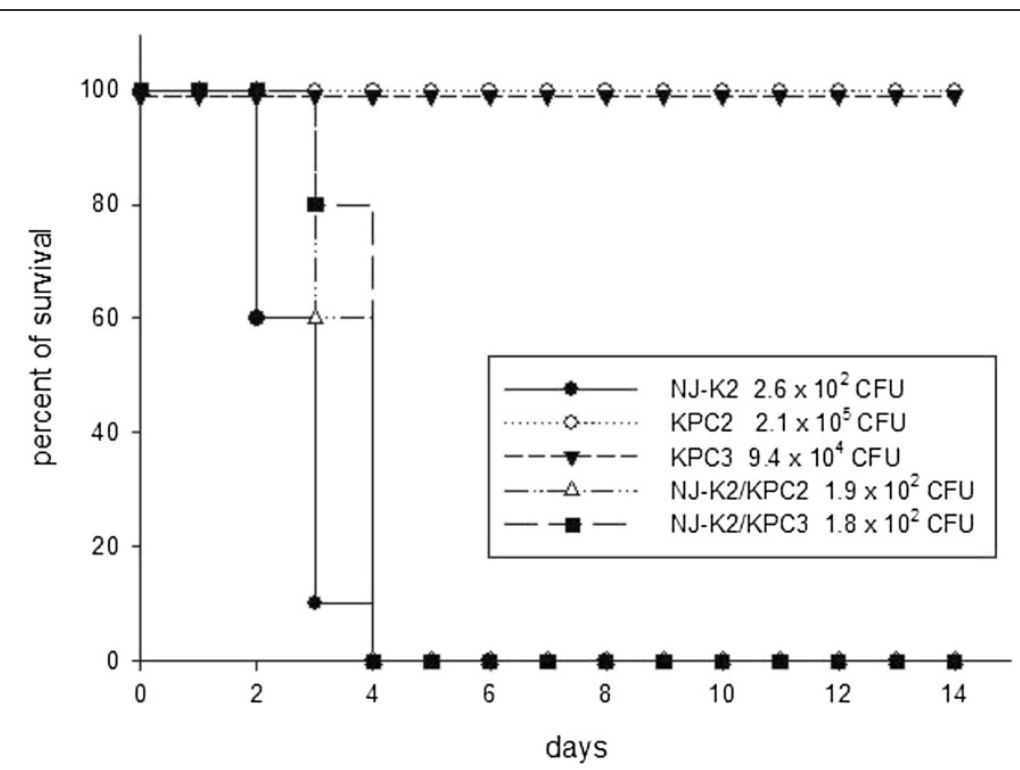

Figure 4 Mice lethality test among Isolates of KPC-2 and -3 , serotype K2 recipient and their transconjugants. The survivals after inoculation of K. pneumoniae were counted over 14 days. Data points represent the percentage of mice surviving in each experimental group over time. ( $n=6$ mice per strain, pooled from two separate experiments). 
cannot be excluded in the future. Efforts to assess the factors that contribute to attributable mortality in KPC K. pneumoniae infections should be continued, including the study of other virulence determinants, antibiotic resistance, host responses, and their relationship with clinical characteristics and outcomes.

\section{Competing interests}

The authors declare that they have no competing interest.

\section{Authors' contributions}

$\mathrm{KS}, \mathrm{DBH}$, and TC made substantial contributions to conception and design, acquisition of data, and analysis and interpretation of data. KS, DBH, and TC were involved in drafting the manuscript, revising it critically for important intellectual content. All authors read and approved the manuscript.

\section{Author details}

${ }^{1}$ National Institute of Infectious Diseases and Vaccinology, National Health Research Institutes, Miaoli, Taiwan. ${ }^{2}$ Graduate Institute of Basic Medical Science, China Medical University, Taichung, Taiwan. ${ }^{3}$ Division of Infectious Diseases, Department of Medicine, Rutgers New Jersey Medical School, Newark, NJ, USA. ${ }^{4}$ Department of Infectious Diseases, Veterans Affairs New Jersey Healthcare System, East Orange, NJ, USA. ${ }^{5}$ Rutgers New Jersey Medical School, 185 South Orange Avenue, Newark, New Jersey 07101, USA.

Received: 3 December 2013 Accepted: 27 March 2014

Published: 31 March 2014

\section{References}

1. Cheng DL, Liu YC, Yen MY, Liu CY, Wang RS: Septic metastatic lesions of pyogenic liver abscess. Their association with Klebsiella pneumoniae bacteremia in diabetic patients. Arch Intern Med 1991, 151(8):1557-1559.

2. Liu YC, Cheng DL, Lin CL: Klebsiella pneumoniae liver abscess associated with septic endophthalmitis. Arch Intern Med 1986, 146(10):1913-1916.

3. Wang JH, Liu YC, Lee SS, Yen MY, Chen YS, Wann SR, Lin HH: Primary liver abscess due to Klebsiella pneumoniae in Taiwan. Clin Infect Dis 1998, 26(6):1434-1438

4. Rivero A, Gomez E, Alland D, Huang DB, Chiang T: K2 serotype Klebsiella pneumoniae causing a liver abscess associated with infective endocarditis. J Clin Microbiol 2010, 48(2):639-641.

5. Ambrozic J, Ostroversnik A, Starcic M, Kuhar I, Grabnar M, Zgur-Bertok D: Escherichia coli ColV plasmid pRK100: genetic organization, stability and conjugal transfer. Microbiology 1998, 144(Pt 2):343-352.

6. D'Agata EM, Gerrits MM, Tang YW, Samore M, Kusters JG: Comparison of pulsed-field gel electrophoresis and amplified fragment-length polymorphism for epidemiological investigations of common nosocomial pathogens. Infect Control Hosp Epidemiol 2001, 22(9):550-554.

7. McDougal LK, Steward CD, Killgore GE, Chaitram JM, MCAllister SK, Tenover FC: Pulsed-field gel electrophoresis typing of oxacillin-resistant Staphylococcus aureus isolates from the United States: establishing a national database. J Clin Microbiol 2003, 41(11):5113-5120.

8. Tenover FC, Arbeit RD, Goering RV, Mickelsen PA, Murray BE, Persing DH, Swaminathan B: Interpreting chromosomal DNA restriction patterns produced by pulsed-field gel electrophoresis: criteria for bacterial strain typing. J Clin Microbiol 1995, 33(9):2233-2239.

9. Bradford PA, Bratu S, Urban C, Visalli M, Mariano N, Landman D, Rahal رل Brooks S, Cebular S, Quale J: Emergence of carbapenem-resistant Klebsiella species possessing the class A carbapenem-hydrolyzing KPC-2 and inhibitor-resistant TEM-30 beta-lactamases in New York City. Clin Infect Dis 2004, 39(1):55-60.

10. Sanger F, Air GM, Barrell BG, Brown NL, Coulson AR, Fiddes CA, Hutchison CA, Slocombe PM, Smith M: Nucleotide sequence of bacteriophage phi X174 DNA. Nature 1977, 265(5596):687-695.

11. Tsai YK, Fung CP, Lin JC, Chen JH, Chang FY, Chen TL, Siu LK: Klebsiella pneumoniae outer membrane porins OmpK35 and OmpK36 play roles in both antimicrobial resistance and virulence. Antimicrob Agents Chemother 2011, 55(4):1485-1493.

12. Siu LK, Ho PL, Yuen KY, Wong SS, Chau PY: Transferable hyperproduction of TEM-1 beta-lactamase in Shigella flexneri due to a point mutation in the pribnow box. Antimicrob Agents Chemother 1997, 41(2):468-470.
13. Wang M, Tran JH, Jacoby GA, Zhang Y, Wang F, Hooper DC: Plasmidmediated quinolone resistance in clinical isolates of Escherichia coli from Shanghai, China. Antimicrob Agents Chemother 2003, 47(7):2242-2248.

14. Yeh KM, Lin JC, Yin FY, Fung CP, Hung HC, Siu LK, Chang FY: Revisiting the importance of virulence determinant magA and its surrounding genes in Klebsiella pneumoniae causing pyogenic liver abscesses: exact role in serotype K1 capsule formation. J Infect Dis 2010, 201(8):1259-1267.

15. Turton JF, Baklan H, Siu LK, Kaufmann ME, Pitt TL: Evaluation of a multiplex PCR for detection of serotypes K1, K2 and K5 in Klebsiella sp. and comparison of isolates within these serotypes. FEMS Microbiol Lett 2008, 284(2):247-252.

16. Chiu SK, Wu TL, Chuang YC, Lin JC, Fung CP, Lu PL, Wang JT, Wang LS, Siu LK, Yeh KM: National surveillance study on carbapenem non-susceptible Klebsiella pneumoniae in Taiwan: the emergence and rapid dissemination of KPC-2 carbapenemase. PLoS One 2013, 8(7):e69428.

17. Siu LK, Fung CP, Chang FY, Lee N, Yeh KM, Koh TH, Ip M: Molecular typing and virulence analysis of serotype K1 Klebsiella pneumoniae strains isolated from liver abscess patients and stool samples from noninfectious subjects in Hong Kong, Singapore, and Taiwan. J Clin Microbiol 2011, 49(11):3761-3765.

18. Turton JF, Englender H, Gabriel SN, Turton SE, Kaufmann ME, Pitt TL: Genetically similar isolates of Klebsiella pneumoniae serotype K1 causing liver abscesses in three continents. J Med Microbiol 2007, 56(Pt 5):593-597.

19. Lin JC, Chang FY, Fung CP, Xu JZ, Cheng HP, Wang JJ, Huang LY, Siu LK: High prevalence of phagocytic-resistant capsular serotypes of Klebsiella pneumoniae in liver abscess. Microbes Infect 2004, 6(13):1191-1198.

20. Yigit H, Queenan AM, Anderson GJ, Domenech-Sanchez A, Biddle JW, Steward CD, Alberti S, Bush K, Tenover FC: Novel carbapenem-hydrolyzing beta-lactamase, KPC-1, from a carbapenem-resistant strain of Klebsiella pneumoniae. Antimicrob Agents Chemother 2001, 45(4):1151-1161.

21. Pournaras S, Zarkotou O, Poulou A, Kristo I, Vrioni G, Themeli-Digalaki K, Tsakris A: A combined disk test for direct differentiation of carbapenemase-producing enterobacteriaceae in surveillance rectal swabs. J Clin Microbiol 2013, 51(9):2986-2990.

22. Siu LK, Yeh KM, Lin JC, Fung CP, Chang FY: Klebsiella pneumoniae liver abscess: a new invasive syndrome. Lancet Infect Dis 2012, 12(11):881-887.

23. Li W, Sun G, Yu Y, Li N, Chen M, Jin R, Jiao Y, Wu H: Increasing occurrence of antimicrobial-resistant hypervirulent (hypermucoviscous) Klebsiella pneumoniae isolates in China. Clin Infect Dis 2014, 58(2):225-232.

doi:10.1186/1471-2334-14-176

Cite this article as: Siu et al:: Plasmid transferability of KPC into a virulent K2 serotype Klebsiella pneumoniae. BMC Infectious Diseases 2014 14:176.

\section{Submit your next manuscript to BioMed Central and take full advantage of:}

- Convenient online submission

- Thorough peer review

- No space constraints or color figure charges

- Immediate publication on acceptance

- Inclusion in PubMed, CAS, Scopus and Google Scholar

- Research which is freely available for redistribution 